\title{
Exploring shear-induced segregation in controlled-velocity granular flows
}

\author{
Lu Jing ${ }^{1}$, Julio M. Ottino ${ }^{1,2,3}$, Richard M. Lueptow ${ }^{1,2,3, *}$, and Paul B. Umbanhowar ${ }^{2}$ \\ ${ }^{1}$ Department of Chemical and Biological Engineering, Northwestern University, Evanston, IL 60208, USA \\ ${ }^{2}$ Department of Mechanical Engineering, Northwestern University, Evanston, IL 60208, USA \\ ${ }^{3}$ Northwestern Institute on Complex Systems (NICO), Northwestern University, Evanston, IL 60208, USA
}

\begin{abstract}
Particle segregation in geophysical and industrial granular flows is typically driven by gravity and shear. While gravity-induced segregation is relatively well understood, shear-induced segregation is not. In particular, what controls segregation in the absence of gravity and the interplay between shear- and gravity-driven segregation remain unclear. Here, we explore the shear-induced segregation force on an intruder particle in controlled-velocity granular flows where the shear profile is systematically varied. The shear-induced segregation force is found to be proportional to the shear rate gradient, which effectively pushes the large intruder from lower to higher shear rate regions. A scaling law is developed for the segregation force that is accurate over a wide range of overburden pressures and shear rates, and hence inertial numbers.
\end{abstract}

\section{Introduction}

Granular materials consisting of different particle species tend to segregate by species size, density, and other properties, which is of great importance in many geophysical and industrial problems $[1,2]$. The understanding and predictive capabilities for segregation in dense granular flows has significantly advanced over the last decades, especially for gravity-driven mechanisms such as kinetic sieving for size segregation and buoyancy for density segregation [35]. However, segregation induced purely by shear (occurring perpendicular to, or in the absence of, gravity) such as in a vertical silo [6] or lateral fault [7], and its significance relative to gravity-related mechanisms, are much less understood.

Shear-induced segregation has previously been associated with the gradient of shear rate [6], shear stress [8], kinetic stress [9], granular temperature [7, 10], and/or solid fraction $[6,11]$. However, a fundamental understanding and general characterization of what drives segregation in the absence of gravity remains elusive. In particular, since the shear profile of a granular flow is typically due to boundary conditions and external forcing, it is difficult to intentionally vary the shear profile for systematic investigation in common flow geometries.

Here, we computationally explore shear-induced segregation using controlled-velocity flows, where a desired velocity profile is imposed via a stabilizing force algorithm [12]. We measure the net segregation force on an intruder particle in various flows with systematically varied shear rate gradients, in the absence of gravity, and therefrom develop a scaling law for shear-induced segregation forces in terms of the shear rate gradient and other flow conditions.

\section{Methods}

\subsection{Velocity-controlled flow}

We simulate controlled-velocity dense granular flows using the open-source discrete element method (DEM) code LIGGGHTS [13]. Controlled-velocity flow has been previously applied to study granular rheology [14-16] and segregation induced by gravity $[12,17,18]$, but it has not been used to explore segregation induced purely by shear.

As shown in Fig. 1(a), bed particles of diameter $d$ and density $\rho$ are sheared in a box with periodic boundaries in the streamwise $(x)$ and spanwise $(y)$ directions, whereas the normal $(z)$ direction is confined by rough walls. A constant overburden pressure $P_{0}$ is applied to the top wall, which is reactive in $z$ and translated in $x$; the bottom wall is stationary. The depthwise profile of the flow velocity $u(z)$ is imposed by applying a stabilizing force (in the $x$ direction) to each particle at each DEM time step [12], and the top wall is translated at a matching speed $u(h)$, where $h$ is the flow thickness. The stabilizing force applied on a particle is $A\left(u\left(z_{p}\right)-u_{p}\right)$, where $z_{p}$ and $u_{p}$ are the instantaneous particle position and velocity, respectively, and $A=0.1 \mathrm{~N} \cdot \mathrm{s} / \mathrm{m}$ (we have verified that varying $A$ by an order of magnitude does not change the results). As shown below, the stabilizing force algorithm effectively maintains a global velocity profile $u(z)$ without modifying the rheology of the flow; that is, particle-particle collisions dominate the granular rheology in the presence of stabilizing forces.

To systematically vary a spatially uniform shear rate gradient, i.e., the curvature of the velocity profile, we specify $u(z)$ as

$$
u(z)=\left(\dot{\gamma}_{0}-\frac{C_{0} h}{2}\right) z+\frac{C_{0}}{2} z^{2},
$$

\footnotetext{
*e-mail: r-lueptow@ northwestern.edu

A video is available at https://doi.org/10.48448/6bkn-ps29
} 
(a)

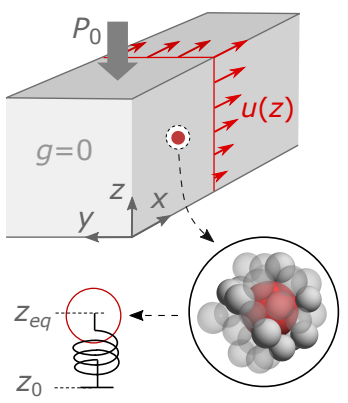

(b)
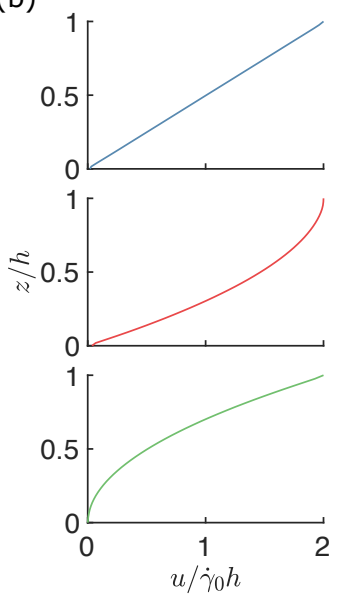
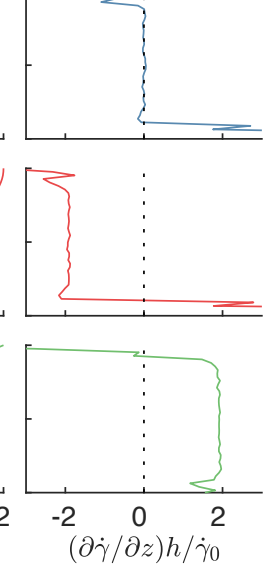

(c)

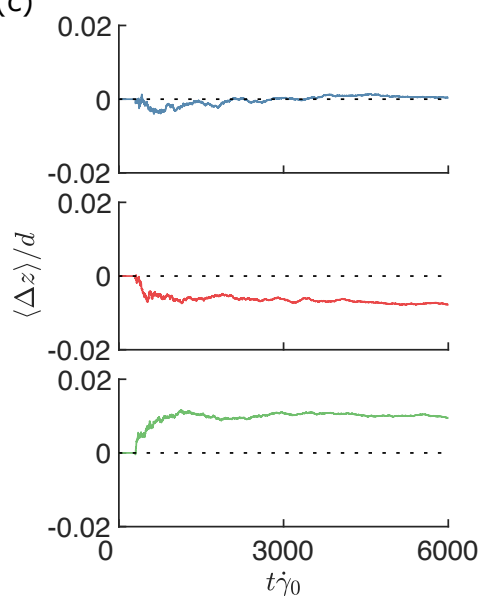

Figure 1. (a) Controlled-velocity granular flow with imposed velocity $u(z)$ and a virtual spring that measures the segregation force on a red intruder particle. Measured (b) kinematics profiles and (c) intruder displacements from DEM simulations for three representative cases $\left(P_{0}=1000 \mathrm{~Pa}, \dot{\gamma}_{0}=30 \mathrm{~s}^{-1}\right)$. Top to bottom: velocity profiles are varied by specifying $C_{0} h / \dot{\gamma}_{0}=\{0,-2,2\}$.

where $\dot{\gamma}_{0}$ and $C_{0}$ are the characteristic shear rate and curvature, respectively, such that

$$
\dot{\gamma}(z)=\dot{\gamma}_{0}-\frac{C_{0} h}{2}+C_{0} z
$$

and

$$
\frac{\partial \dot{\gamma}}{\partial z}=C_{0}
$$

With this form of $u(z)$, a constant shear rate gradient $C_{0}$ is generated, and $C_{0}$ can be varied from negative to positive to manipulate the curvature of the velocity profile $\left(C_{0}=0\right.$ produces a linear profile); see Fig. 1(b). Note that the local shear rate in the middle of the flow (where the intruder particle is placed for segregation force measurement) is $\dot{\gamma}(h / 2)=\dot{\gamma}_{0}$, independent of $C_{0}$, which allows us to control the local flow conditions $\left(P_{0}\right.$ and $\left.\dot{\gamma}_{0}\right)$ around the intruder. Note also that there is no gravity $(g=0)$ in all simulations reported here.

\subsection{Segregation force measurement}

We study shear-induced segregation by determining the net segregation force $F_{\text {seg }}$ acting on an intruder particle (diameter $d_{i}$ ) for various shear rate profiles.

As illustrated in Fig. 1(a), the intruder particle is tethered to a virtual spring that acts only in the $z$-direction $[8,18,19]$. In steady state, the intruder position $z_{t}$ fluctuates about a mean position $z_{e q}=\left\langle z_{t}\right\rangle$ and the spring force balances the segregation force, i.e., $F_{\text {seg }}=k\langle\Delta z\rangle$, where $k$ is the spring stiffness, $\Delta z=z_{t}-z_{0}$ is the intruder displacement, $z_{0}$ is the initial position of the intruder (and the equilibrium position of the spring), and $\langle\cdot\rangle=\int_{0}^{t} \cdot d t$ represents averaging over elapsed time $t$ [see Fig. 1(c)]. The uncertainty in $F_{\text {seg }}$ (error bars) is estimated based on temporally correlated fluctuations in $z_{t}$ [20]. We use a spring stiffness $k=100 \mathrm{~N} / \mathrm{m}$ to ensure a small $\Delta z$, such that $z_{e q}$ and hence the local flow condition are a priori known. It is important to note that, by definition, $F_{\text {seg }}$ is the net contact force (in the $z$-direction) on the intruder exerted by neighboring particles. As such, $F_{\text {seg }}$ alone determines the direction of segregation (no gravitational force is present as $g=0)$.

\subsection{Model parameters}

The primary goal here is to explore how $F_{\text {seg }}$ depends on $\partial \dot{\gamma} / \partial z$, which is varied by specifying $C_{0}$ [see Eq. (3)], for a wide range of flow conditions. Therefore, we vary the dimensionless shear rate gradient $C_{0} h / \dot{\gamma}_{0}$ from -2 to 2 for particular values of $P_{0}$ and $\dot{\gamma}_{0}$ [see Fig. 1(b) for example], and then vary pressure and shear rate $(1000 \leqslant$ $P_{0} \leqslant 3000 \mathrm{~Pa}$ and $10 \leqslant \dot{\gamma}_{0} \leqslant 40 \mathrm{~s}^{-1}$ ) to achieve a wide range of inertial numbers around the intruder, i.e., $I\left(z_{e q}\right)=\dot{\gamma}\left(z_{e q}\right) d \sqrt{\rho / p\left(z_{e q}\right)}$, ranging from 0.05 to 0.44 .

Other parameters are kept constant in all simulations, including the domain size $(30 d, 20 d, 30 d)$ and the intruderbed particle size ratio $R=d_{i} / d=2$. Note that $R$ is a key parameter controlling gravity-driven segregation [18]. However, the influence of $R$ on shear-induced segregation will be explored in separate work [21]. Particle contact forces are solved using the Hertz model, and particle properties are identical to those in [18]: $d=5 \mathrm{~mm}$ (with a uniform $10 \%$ polydispersity), $\rho=2500 \mathrm{~kg} / \mathrm{m}^{3}$, Young's modulus is $5 \times 10^{7} \mathrm{~Pa}$, Poisson's ratio is 0.4 , the coefficient of restitution is 0.8 , and the coefficient of friction is 0.5 .

\section{Results and discussion}

\subsection{Intruder displacement}

For a representative set of $P_{0}$ and $\dot{\gamma}_{0}$, three values of $C_{0}$ are considered in Fig. 1(b-c) to demonstrate the influence of velocity profiles on the intruder displacement. For a linear velocity profile $(\partial \dot{\gamma} / \partial z=0)$, the intruder position 

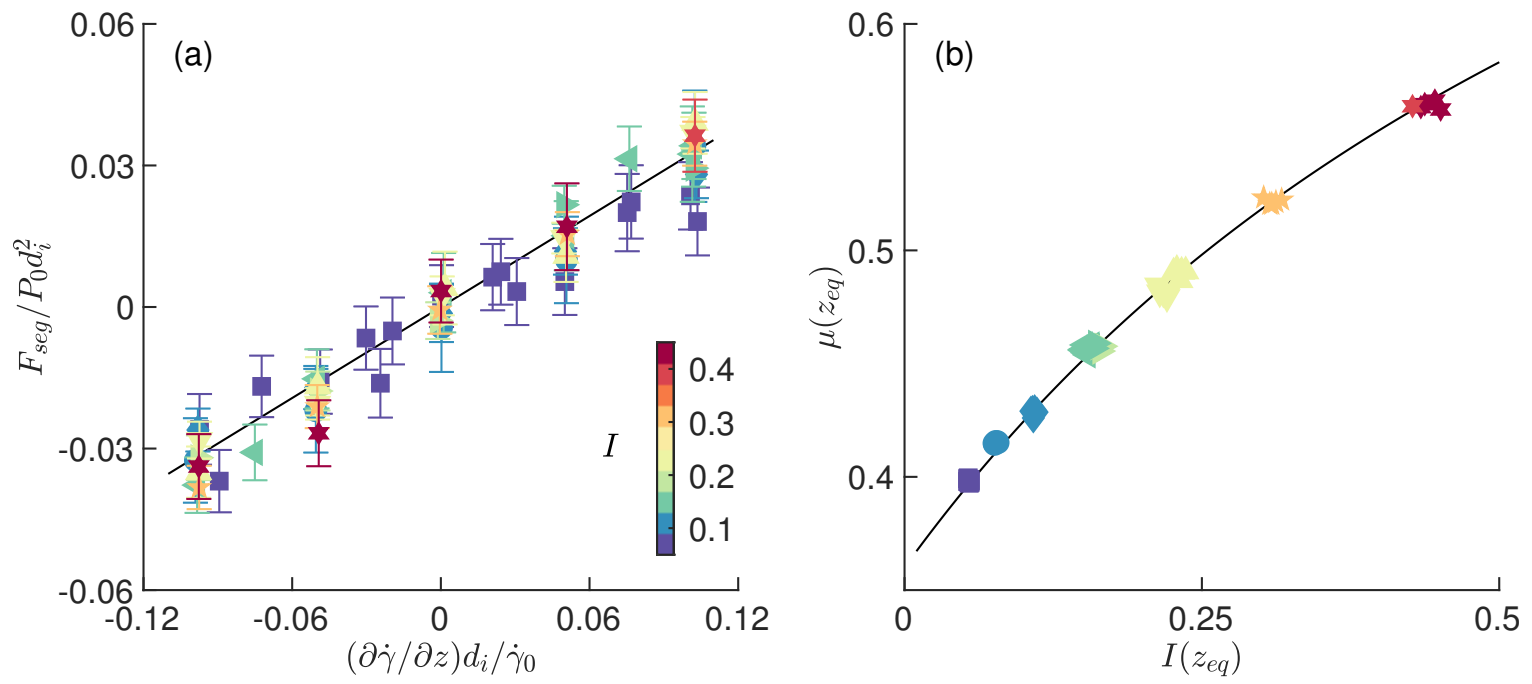

Figure 2. (a) Normalized segregation force vs. normalized shear rate gradient. Local inertial numbers $I\left(z_{\text {eq }}\right)$ ranging from 0.05 to 0.44 (color bar) are generated with $1000 \leqslant P_{0} \leqslant 3000 \mathrm{~Pa}$ and $10 \leqslant \dot{\gamma}_{0} \leqslant 40 \mathrm{~s}^{-1}$. For for each combination of $P_{0}$ and $\dot{\gamma}_{0}, C_{0} h / \dot{\gamma}_{0}$ is varied from -2 to 2 to generate negative to positive curvatures $\partial \dot{\gamma} / \partial z$ [see Eq. (3)]. The slope of the linear fit through the origin is 0.32 . (b) Local $\mu(I)$ data at $z=z_{\text {eq }}$. The curve represents $\mu(I)=0.36+(0.94-0.36) /(0.8 / I+1)$, which is obtained using simple shear simulations.

fluctuates about the initial depth, $\langle\Delta z\rangle \approx 0$, indicating that there is no net segregation force for a uniform shear. Although expected by symmetry, it is interesting to note that the gradient of velocity (i.e., the shear rate) alone does not drive segregation. For a concave-up velocity profile $(\partial \dot{\gamma} / \partial z<0)$, the intruder moves toward negative $z$, i.e., $\langle\Delta z\rangle<0$. Likewise, for a concave-down velocity profile $(\partial \dot{\gamma} / \partial z>0)$ the intruder moves toward positive $z$, i.e., $\langle\Delta z\rangle>0$. These results confirm that a non-zero shear rate gradient (or, equivalently, a non-linear velocity profile) can indeed drive size segregation, and the large intruder always migrates from lower to higher shear rate regions, consistent with the direction of shear-driven segregation in dense silo flows [6].

\subsection{Segregation forces}

Intruder displacement is driven by the net segregation force $F_{\text {seg }}$, which depends on the shear rate gradient $\partial \dot{\gamma} / \partial z$. Indeed, using $P_{0} d_{i}^{2}$ and $\dot{\gamma}_{0} / d_{i}$ to normalize $F_{\text {seg }}$ and $\partial \dot{\gamma} / \partial z$, respectively, Fig. 2(a) shows that $F_{\text {seg }}$ is proportional to $\partial \dot{\gamma} / \partial z$ for $C_{0} h / \dot{\gamma}_{0}$ varied from -2 to 2 for various combinations of $P_{0}$ and $\dot{\gamma}_{0}$ (hence various $I$; see color bar). Remarkably, all results collapse for $I$ ranging from 0.05 to 0.44 . Figure 2(b) shows the rheological data measured at $z_{e q}$, around which the intruder fluctuates, for all simulations. The data fall on a reference $\mu(I)$ curve that we obtain for simple shear DEM simulations (uncontrolled and with no intruder) for the same particle properties we use for controlled-velocity flows. This agreement indicates that, as noted above, applying stabilizing forces to control the velocity profile does not affect the rheology of the granular flow.

Based on the data collapse shown in Fig. 2(a), we propose a scaling law for shear-induced segregation forces:

$$
F_{\text {seg }} \propto \frac{p}{\dot{\gamma}} \frac{\partial \dot{\gamma}}{\partial z} d_{i}^{3}
$$

where $p, \dot{\gamma}$, and $\partial \dot{\gamma} / \partial z$ are local flow conditions around the intruder, and the constant of proportionality is insensitive to $I$ at least for the range we explore $(0.05 \leqslant I \leqslant 0.44)$. Note that we have verified that the particle size, but not the system size, is the relevant length scale to collapse the data in Fig. 2(a).

\section{Conclusion}

Using controlled-velocity dense granular flows, in the absence of gravity, we explore segregation induced purely by shear and measure the net segregation force on an intruder particle. The segregation force is proportional to the shear rate gradient, which drives large particles from lower to higher shear rate regions. Based on dimensional analysis, a scaling law is developed for the shear-induced segregation force, which is valid for a wide range of overburden pressures, shear rates, and inertial numbers. In work being prepared for publication [21], effects of the size ratio and density ratio between intruder and bed particles are studied, and the relevance of the proposed scaling law is tested in more general flow configurations where shearand gravity-induced mechanisms are both present.

\section{Acknowledgments}

We thank Yi Fan and John Hecht for valuable discussions. This material is based upon work supported by the National Science Foundation under Grant No. CBET1929265. 


\section{References}

[1] S.B. Savage, C.K.K. Lun, J. Fluid Mech. 189, 311 (1988)

[2] J.M. Ottino, D.V. Khakhar, Annu. Rev. Fluid Mech. 32, 55 (2000)

[3] D.R. Tunuguntla, T. Weinhart, A.R. Thornton, Comput. Part. Mech. 4, 1 (2016)

[4] J.M.N.T. Gray, Annu. Rev. Fluid Mech. 50, 407 (2018)

[5] P.B. Umbanhowar, R.M. Lueptow, J.M. Ottino, Annu. Rev. Chem. Biomol. Eng. 10, 5.1 (2019)

[6] Y. Fan, K.M. Hill, Phys. Rev. Lett. 106, 218301 (2011)

[7] R. Itoh, T. Hatano, Philos. T. R. Soc. A 377, 20170390 (2019)

[8] F. Guillard, Y. Forterre, O. Pouliquen, J. Fluid Mech. 807, R1 (2016)

[9] Y. Fan, K.M. Hill, New J. Phys. 13, 095009 (2011)

[10] K.M. Hill, D.S. Tan, J. Fluid Mech. 756, 54 (2014)

[11] K.M. Hill, Y. Fan, Phys. Rev. Lett. 101, 088001 (2008)
[12] A.M. Fry, P.B. Umbanhowar, J.M. Ottino, R.M. Lueptow, Phys. Rev. E 97, 062906 (2018)

[13] C. Kloss, C. Goniva, A. Hager, S. Amberger, S. Pirker, Prog. Comput. Fluid Dy. 12, 140 (2012)

[14] E. Lerner, G. Düring, M. Wyart, Proc. Natl. Acad. Sci. U.S.A. 109, 4798 (2012)

[15] A.H. Clark, J.D. Thompson, M.D. Shattuck, N.T. Ouellette, C.S. O’Hern, Phys. Rev. E 97, 062901 (2018)

[16] K. Saitoh, B.P. Tighe, Phys. Rev. Lett. 122, 188001 (2019)

[17] Y. Duan, P.B. Umbanhowar, J.M. Ottino, R.M. Lueptow, Phys. Rev. Fluids 5, 044301 (2020)

[18] L. Jing, J.M. Ottino, R.M. Lueptow, P.B. Umbanhowar, Phys. Rev. Res. 2, 022069 (2020)

[19] K. van der Vaart, M.P. van Schrojenstein Lantman, T. Weinhart, S. Luding, C. Ancey, A.R. Thornton, Phys. Rev. Fluids 3, 074303 (2018)

[20] N.F. Zhang, Metrologia 43, S276 (2006)

[21] L. Jing, J.M. Ottino, R.M. Lueptow, P.B. Umbanhowar, https://arxiv.org/abs/2103.09386 (2021) 